Article

\title{
Strengthening the Competitiveness and Sustainability of a Semiconductor Manufacturer with Cloud Manufacturing
}

\section{Toly Chen}

Department of Industrial Engineering and Systems Management, Feng Chia University, 100, Wenhwa Road, Seatwen, Taichung City 407, Taiwan; E-Mail: tolychen@ms37.hinet.net; Tel.: +886-4-24517250; Fax: +886-4-24510240

Received: 11 November 2013; in revised version: 13 December 2013 / Accepted: 27 December 2013 / Published: 3 January 2014

\begin{abstract}
Cloud manufacturing (CMfg) is a new-generation service-oriented networked manufacturing model that provides distributed users centralized managed manufacturing resources, ability, and services. CMfg is applied here to a semiconductor manufacturing factory. Benefits are classified into five aspects: cost savings, efficiency, additional data analysis capabilities, flexibility, and closer partner relationships. A strength, weakness, opportunity, and threat (SWOT) analysis is done which guides a semiconductor manufacturer in planning CMfg implementation projects. Simulation of a wafer fabrication factory (wafer fab) is used as an example. Several CMfg services are proposed for assisting the fab simulation activities through the collaboration of cloud service providers, software vendors, equipment suppliers, and the wafer fab. The connection with the competitiveness and sustainability of a wafer fab is also stressed.
\end{abstract}

Keywords: cloud manufacturing; cloud computing; semiconductor; wafer fabrication; competitiveness; sustainability

\section{Introduction}

The competition in the semiconductor industry, especially the dynamic random access memory (DRAM) industry, is becoming fiercer. It is difficult to survive in this industry. Some events occurred recently highlighted this fact:

(1) In December 2008, the Taiwan DRAM industry was on the verge of bankruptcy. The Ministry of Economic Affairs of Taiwan established the "DRAM Industry Task Force" to help Taiwan DRAM makers embark on organizational reengineering. 
(2) In January 2009, Germany DRAM maker Qimonda declared bankruptcy.

(3) In February 2012, Japanese DRAM maker Elpida filed for bankruptcy protection.

It is even difficult to gain and maintain a competitive edge in the semiconductor industry. In order to enhance the global competitiveness of the European semiconductor industry, in 2009, a number of world-renowned European semiconductor companies executed the "implementing manufacturing science solutions to increase equipment productivity and fab performance (IMPROVE)" project. The target was to enhance the efficiency of semiconductor manufacturing while reducing costs and shortening the cycle time [1]. In order to seek technological breakthroughs and industry-leading, the world's largest foundry, Taiwan Semiconductor Manufacturing Company (TSMC), announced that they will invest 12 billion dollars in capital expenditures in 2014 [2].

On the other hand, the trend of automation and unmanned operations in semiconductor manufacturing is becoming more obvious. For example, in Plant \#14 of TSMC, there is no operator, but only machines that are neatly arranged and operated automatically [2]. In addition, the trend of manufacturing as a service (MaaS) is increasingly evident. To meet these emerging needs, cloud manufacturing (CMfg or $\mathrm{CM}$ ) and services are considered to be viable options. CMfg and services are derived from cloud computing and technologies. According to Mell and Grance [3], cloud computing is a model for enabling ubiquitous, convenient, on-demand network access to a shared pool of configurable computing resources (e.g., networks, servers, storages, applications, and services) that can be rapidly provisioned and released with minimal management efforts or service provider interaction. As a direct extension in the manufacturing sector, $\mathrm{CMfg}$ can be defined as a model for enabling ubiquitous, convenient, on-demand network access to a shared pool of configurable manufacturing resources (e.g., software tools, equipments, and manufacturing capabilities) that can be rapidly provisioned and released with minimal management efforts or service provider interaction $[4,5]$. According to Zhang et al.'s view, CMfg is a new-generation service-oriented networked manufacturing model that can provide users distributed in different places with manufacturing resources and manufacturing ability services through centralized management [6]. However, whether the concept of cloud computing can be fully applied to manufacturing is a problem. For example, Fan et al. [7] compared the differences between information services and manufacturing services:

(1) Interaction: Manufacturing services usually rely on the interaction between users and services.

(2) Real time: Manufacturing services must reflect the actual status of the equipment or design unit, and be able to respond to customer requests in a timely manner.

(3) Cooperation: A variety of manufacturing services should be integrated to meet customer demand in a cooperative manner, i.e., manufacturing service composition.

(4) Longer life cycle: Compared with information services, the life cycle of a manufacturing service may be longer.

(5) Data-intensive: Compared to information services, manufacturing services often need to transfer or analyze a large amount of data.

(6) Complicated functionality and infrastructure: The functionality of a manufacturing service, and the infrastructure for such a functionality, is often more complicated than expected, especially in a semiconductor manufacturing factory. 
(7) Highly specialized: The degree of specialization of manufacturing services is high; information technology is only a small part.

(8) Expertise-based: Compared to information services that are usually based on data, manufacturing services are usually based on particular expertise.

In addition, there are three roles in cloud computing: users, application providers, and physical resource providers [8]. A factory (individual or original engineering manufacturer (OEM)) is typically a user in CMfg. However, if a factory provides its capacity to others to rent, it becomes a physical resource provider. In other words, a factory can play more than one role in CMfg. A comprehensive review on CMfg can be seen in Wu et al. [8].

Nevertheless, from the existing reports and case studies, the benefits of CMfg appear in the following respects: cost savings, efficiency, additional data analysis capabilities, flexibility, and closer partner relationships (see Figure 1). For a manufacturer, customer relationship management (CRM) is an important business function that has been put into clouds to streamline the workflow and communicate with buyers [9]. In addition, enterprise resource planning (ERP) is a management information system used to coordinate the various business functions of a manufacturer. Several ERP system vendors, such as Epicor [10], have developed cloud services so that a manufacturer can still manage the same business functions without the need to purchase an ERP system. The advantages of a cloud ERP (or web-based ERP) system over the traditional ERP systems include faster implementation, no hardware or software costs to upgrade or maintain the system, and low usage costs. These transaction or management systems vendors usually supply decision support systems as well. Therefore, due to the cost savings of CMfg applications, a semiconductor manufacturer can more easily obtain additional analysis capabilities. Further, CMfg has been widely applied in the machine tool industry to diagnose a malfunctioning machine tool via the Internet and suggest possible repair treatments. That is very useful if a future wafer fabrication factory (wafer fab) will be fully automated. Two important concepts in cloud computing - interoperability and scalability - if successfully applied to CMfg, can contribute to the flexibility of a semiconductor manufacturer in responding to ordering requests and in adjusting the factory capacity.

Figure 1. The benefits of cloud manufacturing (CMfg) (Please amend the typo "Cost Savings" in the picture.).

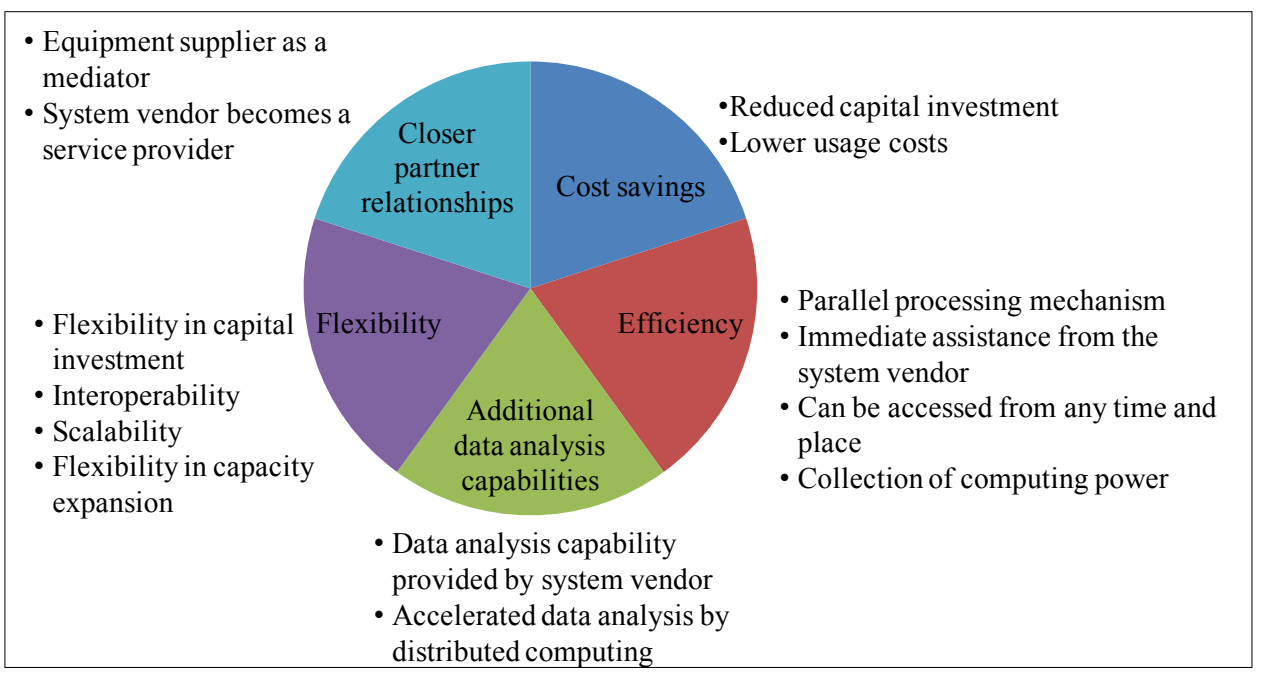


However, it is questioned that CMfg may be suitable only for small or mid-sized enterprises [10]. A semiconductor manufacturer, as a capital-intense business, is not hesitant to buy all the necessary systems or equipment. That makes some of the cost-saving incentives insignificant. In addition, Davidson [11] mentioned that there has been a notable hesitancy in manufacturing operations management to migrate systems to clouds. Possible reasons include:

(1) Manufacturing operations management people are conservative when it comes to change.

(2) Internet connection is sometimes intermittent; that is not conducive to the continuous operations in manufacturing.

(3) Decision makers are highly concerned about the safety of their manufacturing data on a public cloud.

For these reasons, the objective of this study is to assess the feasibility of applying CMfg technologies to strengthening the competitiveness and sustainability of a semiconductor manufacturer. To this end, the following procedure is followed. First of all, a strength, weakness, opportunity, and threat (SWOT) analysis is conducted for the project "applying CMfg technologies to strengthen the competitiveness and sustainability of a semiconductor manufacturer." Subsequently, wafer fab simulation as a cloud service is taken as an example to explore how $\mathrm{CMfg}$ can improve the effectiveness of managing a wafer fab. Finally, this study is concluded and some directions for future research are given.

\section{Methodology}

\subsection{SWOT Analysis}

SWOT analysis/matrix is a structured planning method used to evaluate the strengths, weaknesses, opportunities, and threats involved in a project or in a business venture [12]. In this study, the project "applying CMfg technologies to strengthen the competitiveness and sustainability of a semiconductor manufacturer" is to be evaluated. A SWOT analysis can be carried out for a product, place, industry or person. It involves specifying the objective of the business venture or project and identifying the internal and external factors that are favorable and unfavorable to achieving that objective. In this project, the objective is "to strengthen the competitiveness and sustainability of a semiconductor manufacturer".

First, to achieve the objective, a semiconductor manufacturer has the following strengths:

(1) The capital adequacy of a semiconductor manufacturer is very high.

(2) The degree of automation of machinery and equipments in a semiconductor manufacturing factory is very high.

(3) The system and information technology-related professionals are not lacking.

However, a semiconductor manufacturer is subject to the following weaknesses that are not conducive to the achievement of the objective:

(1) Since the capital adequacy of a semiconductor manufacturer is very high, it seems there is no need to rent an equipment or information system.

(2) To a semiconductor manufacturer, the focus of cost savings is always on yield improvement (e.g., [13,14]), cost learning (e.g., [15]), and cycle time reduction (e.g., [16]), but never on the reduction of investing in equipment or information systems. 
(3) Many production and operational data have a high degree of confidentiality, so they may not be suitable to be transmitted through the the Internet.

(4) There are many heterogeneous and non-integrated business and shop floor planning systems in a semiconductor manufacturing factory [17], so it becomes difficult to provide unified protocols for the use of manufacturing resources [7].

Nevertheless, the following events provided several opportunities to the existing semiconductor manufacturers:

(1) The emergence of advanced manufacturing technologies, such as lean manufacturing (LM), cloud manufacturing (CMfg), manufacturing grid (MGrid), global manufacturing (GM), virtual manufacturing (VM), and agile manufacturing (AM). The definitions of these items are given in Table 1.

(2) Cloud computing is widely applied.

(3) Semiconductor manufacturers are seeking new ways to enhance their competitiveness and sustainability.

(4) Most of the existing CMfg technologies are from the view of information technology, such as $\mathrm{Xu}$ [5] and $\mathrm{Wu}$ et al. [8], rather than from a manufacturing point of view. In other words, semiconductor manufacturers need manufacturing-oriented $\mathrm{CMfg}$, supplemented by information technology. While information technology-oriented CMfg can be seen as the re-allocation of information technology resources of a manufacturer, such as ERP or CRM as a cloud service, manufacturing-oriented CMfg can be considered as "the re-allocation of manufacturing resources with the aid of cloud computing technologies". For example, the scalability of a factory is the flexibility in expanding the capacity that is equivalent to the ease of re-allocating equipments from the suppliers to the factory. In addition, a bottleneck in a factory is a machine which capacity is allocated to many lots within the same time interval. To eliminate the bottleneck, other machines should be re-allocated to these lots instead somehow. To this end, factory simulation is a useful tool and can be enhanced with cloud computing technologies.

(5) The popularity of smartphones increases the demand for semiconductors.

(6) According to LNS Manufacturing Operations Management Survey, only 7\% of respondents currently use cloud technology. However, $17 \%$ of respondents replied that they are planning to use cloud/SaaS (software as a service) based solutions for manufacturing operations management applications in the future [11].

In the meantime, semiconductor manufacturers are also facing the following threats:

(1) The rise of the Chinese market, and of its manufacturers, brings opportunities and threats to existing firms.

(2) There is a decline in the average selling price (ASP).

(3) The advances in wafer fabrication processes turn into a cost advantage over the followers.

(4) The huge investment in advanced fabrication processes will expand the advantage in cost, yield, production capacity, and other aspects in the future. 
Table 1. The definitions of some advanced manufacturing technologies.

\begin{tabular}{ll}
\hline Advanced Manufacturing Technology & Definition \\
\hline Lean manufacturing (LM) & $\begin{array}{l}\text { Lean manufacturing (LM) refers to a business concept } \\
\text { wherein the goal is to minimize the amount of time and } \\
\text { resources used in the manufacturing processes and other } \\
\text { activities of an enterprise, with emphasis on eliminating } \\
\text { all forms of wastage [18]. }\end{array}$ \\
\hline Manufacturing grid (MGrid) is a supportive environment \\
to facilitate the sharing and integration of manufacturing \\
resources by encapsulating and integrating design, \\
manufacturing, management, information technology, \\
and knowledge resources distributed in \\
different regions or enterprises [7].
\end{tabular}

A summary of the SWOT analysis results is presented in Figure 2.

Figure 2. A summary of the strength, weakness, opportunity and threat (SWOT) analysis results.

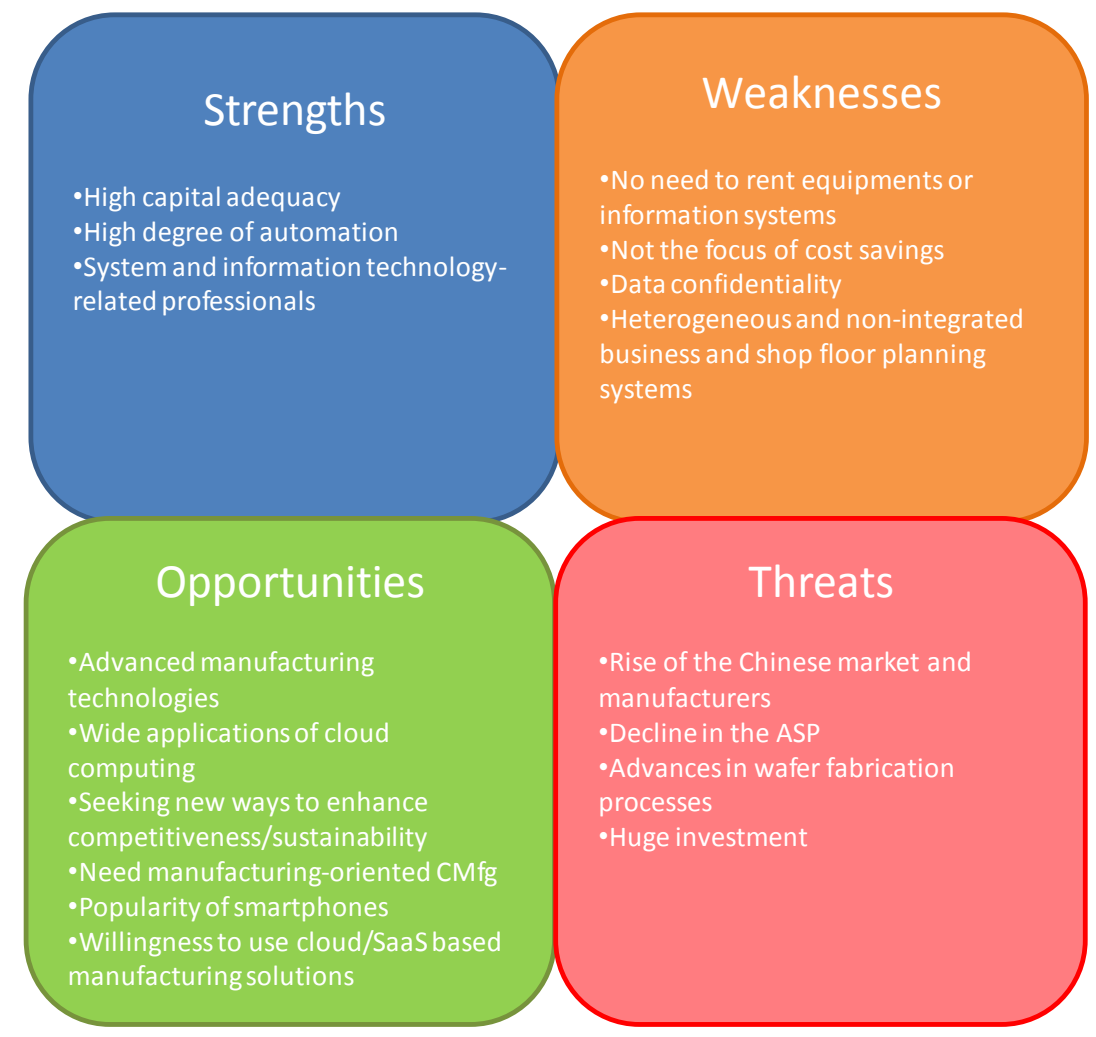




\section{CMfg Applications for Fab Simulation}

Wu et al. [8] listed five issues for future CMfg investigation, including high speed, long distance industrial control systems, flexibility enablement, business models, cloud computing applications in manufacturing, and prominent implementation architectures. From the manufacturing view of CMfg, eliminating the bottleneck of a wafer fab by re-allocating machines is a feasible direction. To this end, fab simulation is useful and can be enhanced with cloud computing technologies. In this study, cloud computing applications to fab simulation are to be investigated. Such applications can also increase the speed and flexibility of fab simulation (see Figure 3). Based on the concept of resource service composition (RSC), a prominent implement architecture is also established.

Figure 3. CMfg issues and the scope of this study.

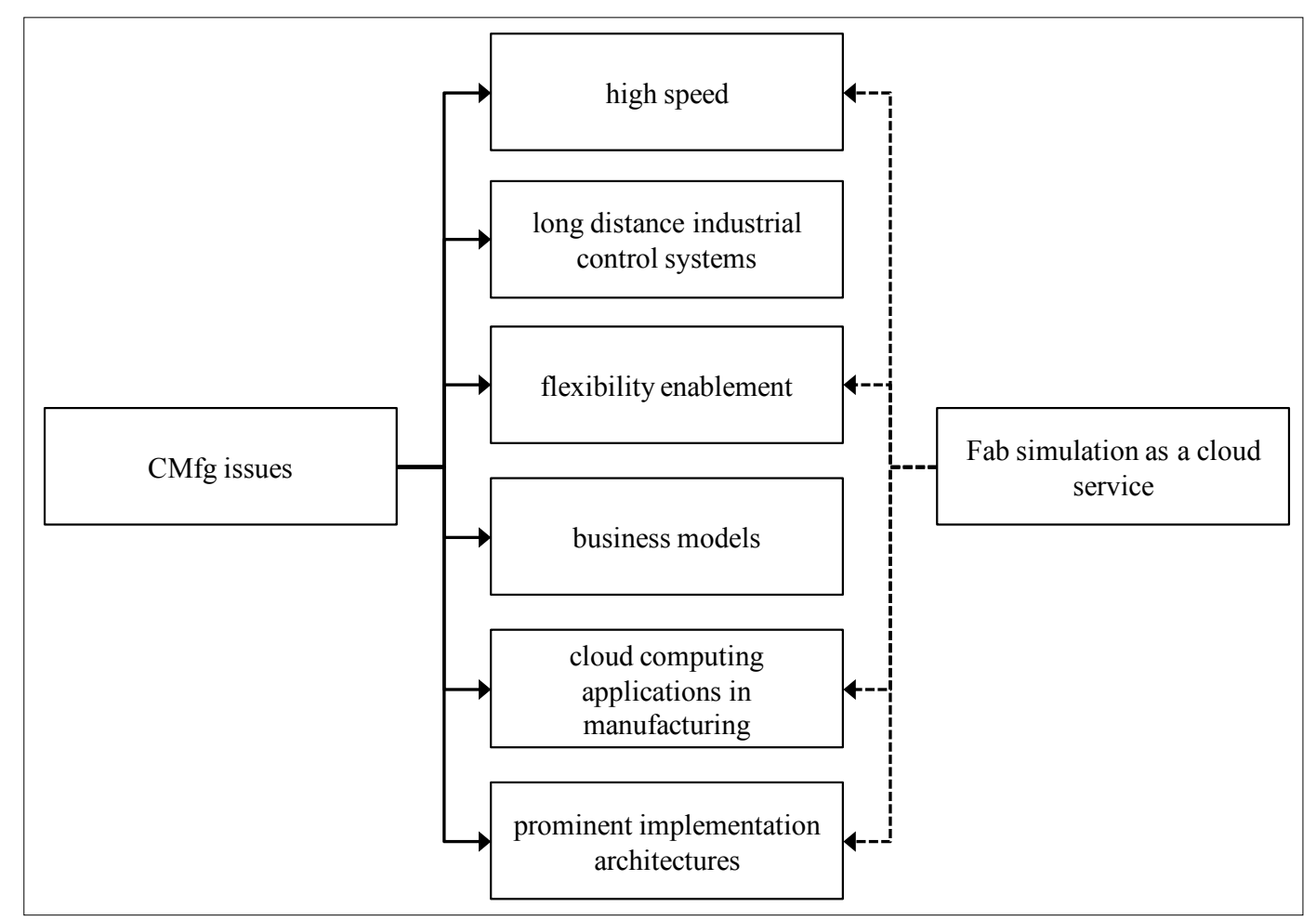

\subsection{Fab Simulation as a Cloud Service (FSaaCS)}

A wafer fab is a very large and complex system usually involving thousands of lots and hundreds of machines. Simulation of such a system is a daunting task. In addition, it often takes several hours to run a single replication of fab simulation for a horizon of a few months. However, this approach is obviously insufficient, taking into account the uncertainty and human intervention inherent in the wafer fabrication process. Theoretically, at least hundreds of simulation replications need to be run to obtain the average and standard deviation of the results, which causes a great burden on the production management personnel. Therefore, fab simulation systems, no wonder are often used in academic research, but rarely in guiding real-time operations of a wafer fab.

The procedure for simulating a wafer fab is illustrated in Figure 4. Among them, CMfg can play a role in at least the following aspects: 
(1) Preparing and analyzing data: Cloud service providers use their statistical analysis software to provide this functionality in response to a user's request, i.e., the so-called statistical analysis as a cloud service (SAaCS). Owing to the huge data, a user is suggested to upload an extensible markup language (XML) data file to be analyzed (see Figure 5) through a Web-based interface. The results are the corresponding probability distributions, regression equations, forecasts, or hypothesis testing results represented in a standard terminology across different cloud services and providers.

(2) Defining equipments: Although visualization may not be so important for simulating a wafer fab, critical components to visualize a fab simulation model are the specifications, pictures, and other basic data of equipments that can be referenced from a pool maintained collaboratively by the equipment suppliers, i.e., the so-called equipment definition as a cloud service (EDaaCS), while some specifications of equipments may be customized and therefore are not publicly available data and should be uploaded in the form of an XML file (see Figure 6) by the wafer fab. The results of SAaaCS become an input to EDaaCS. Later on, a cloud service provider can build the layout on behalf of a wafer fab according to the equipment data with the assistance of a layout software vendor. The concept of EDaaCS is illustrated in Figure 7.

Figure 4. The procedure for simulating a wafer fab.

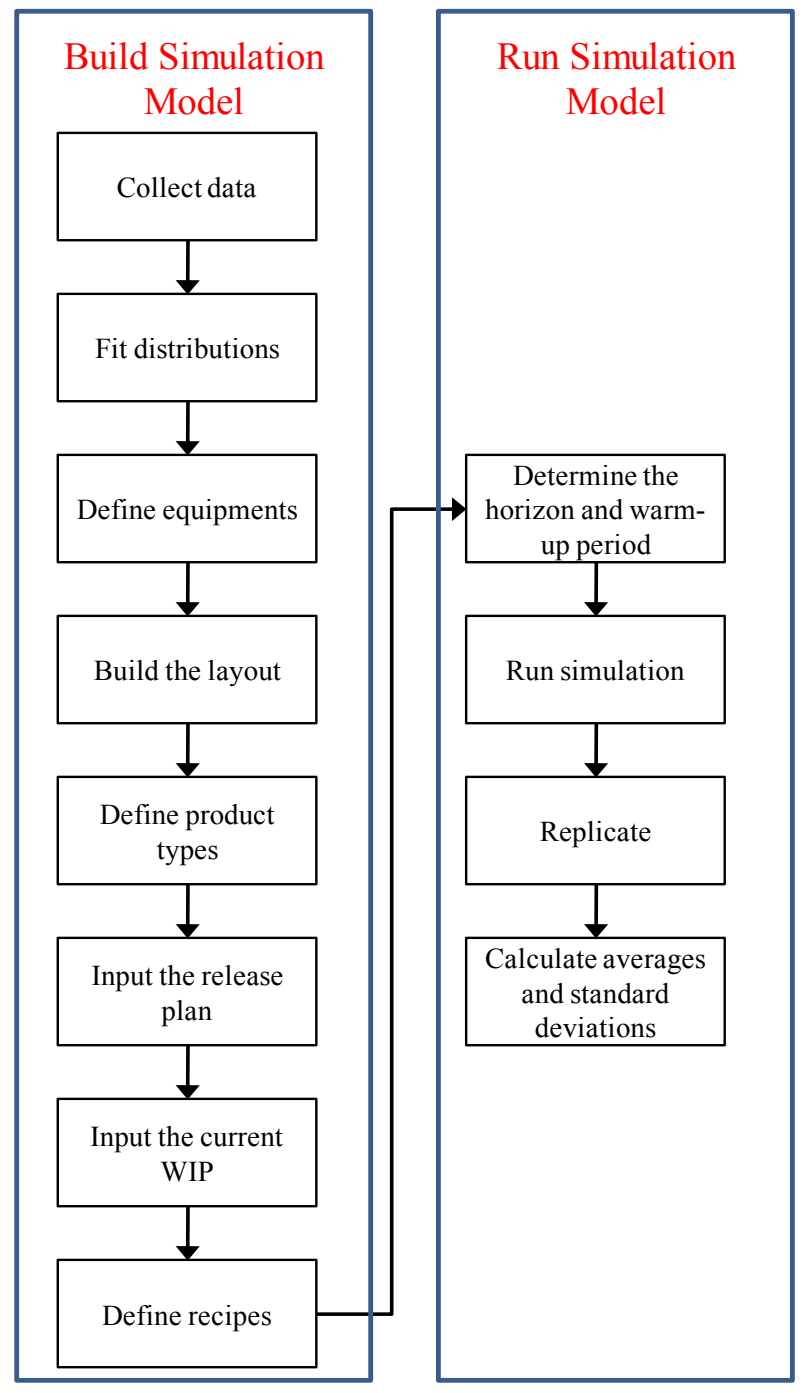


Figure 5. An example XML file for statistical analysis as a cloud service (SAaaCS).

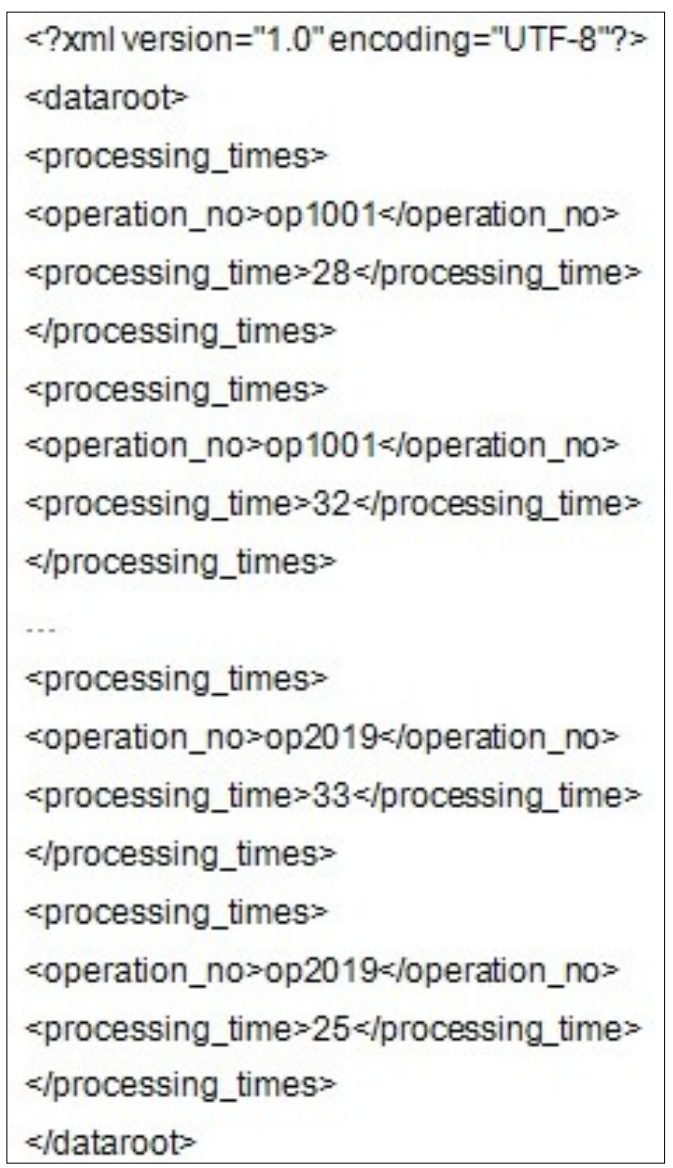

Figure 6. An example XML file for equipment definition as a cloud service (EDaaCS).

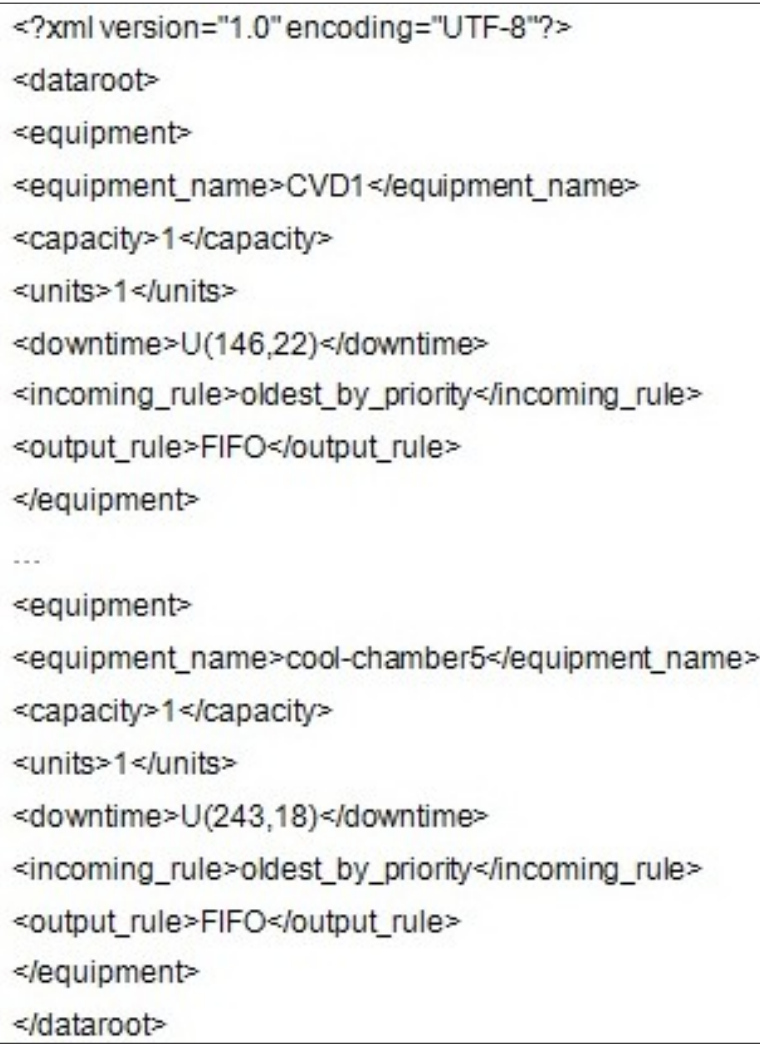


Figure 7. The concept of EDaaCS.

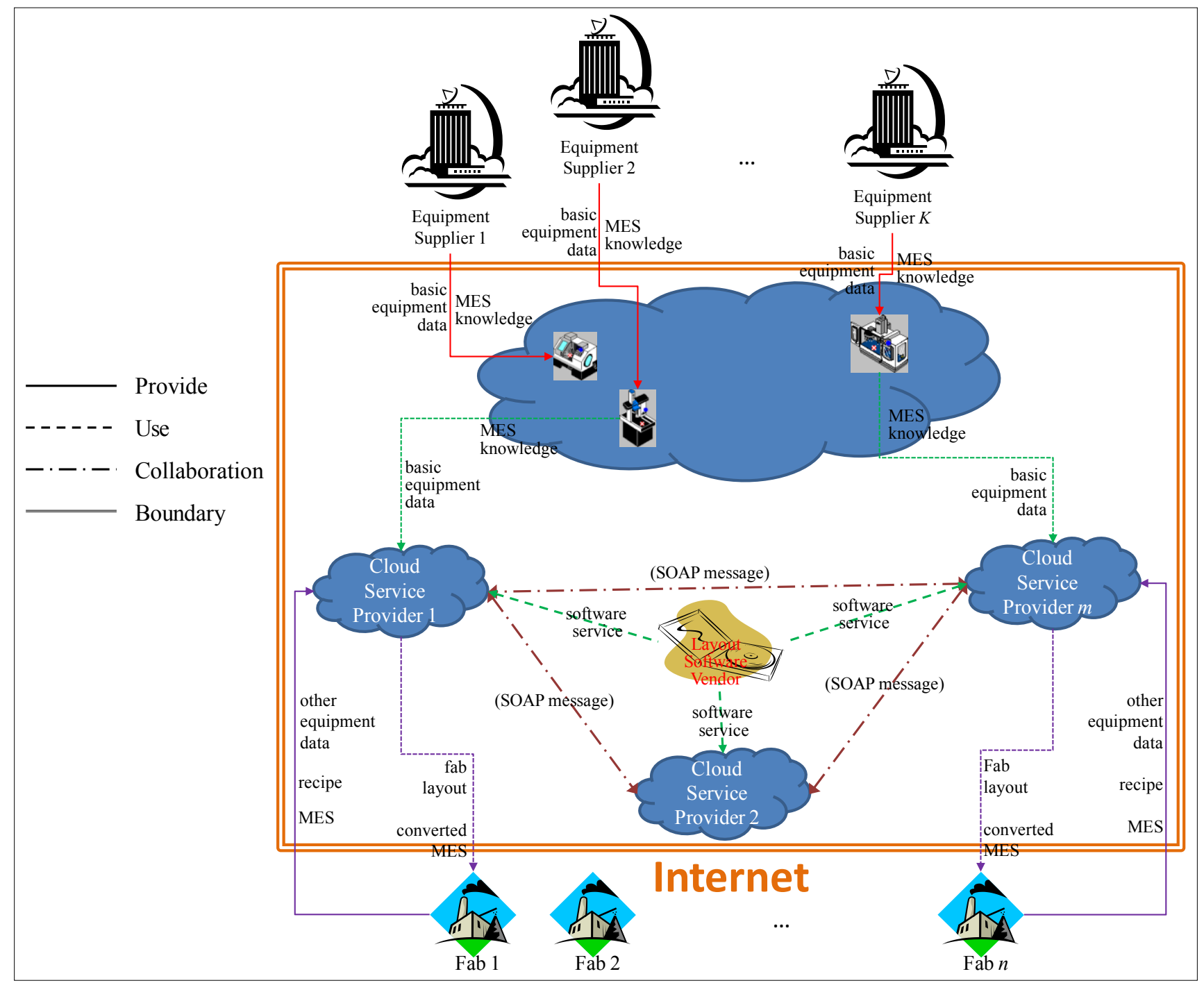

(3) Building the layout: Cloud services, such as Autodesk 360 Cloud Services, have been designed for facility layout, i.e., the so-called facility layout as cloud service (FLaaCS). Establishing the layout of a wafer fab is a laborious and time-consuming task. Fortunately, it is usually done once when a new wafer fab is being built. Later on, only maintenance (minor adjustment) will be needed. Since equipment specifications are an important factor to facility layout, the cloud service EDaaCS can contribute to the efficiency of laying out a wafer fab. In addition, if the capacity of a wafer fab is to be expanded, a major modification will need to be made to the fab layout. At this time, an easy-to-modify fab layout would be useful. That depends on specific computation intelligence, and should be supported by the software vendor as a FLaaCS. In other words, FLaaCS is important to the scalability of a wafer fab (see Figure 8). 
Figure 8. The relationships among EDaaCS, facility layout as cloud service (FLaaCS), and scalability.

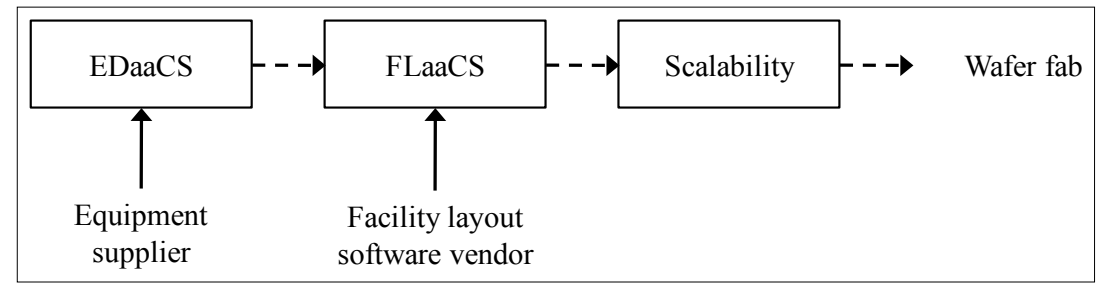

(4) Defining and converting recipes: In standalone fab simulation systems, the formats for recording recipes are usually different from system to system. In addition, some of these systems operate on the basis of sequential operations, while others are object-oriented. Further, some programs embedded into the operations may be coded in different languages. These difficulties have to be tackled in designing a cloud service for defining recipes, i.e., defining recipes as a cloud service (DRaCS). An example XML file for defining a recipe is given in Figure 9. In addition, a cloud service provider can also help in defining the recipe of manufacturing a specific product, or in converting recipes or manufacturing execution systems (MES) with the aid of equipment suppliers, i.e., the so-called recipe conversion as a cloud service (RCaaCS), also shown in Figure 7. That is considered as an essential step to the interoperability of a wafer fab.

Figure 9. An example XML file for defining recipes as a cloud service (DRaaCS).

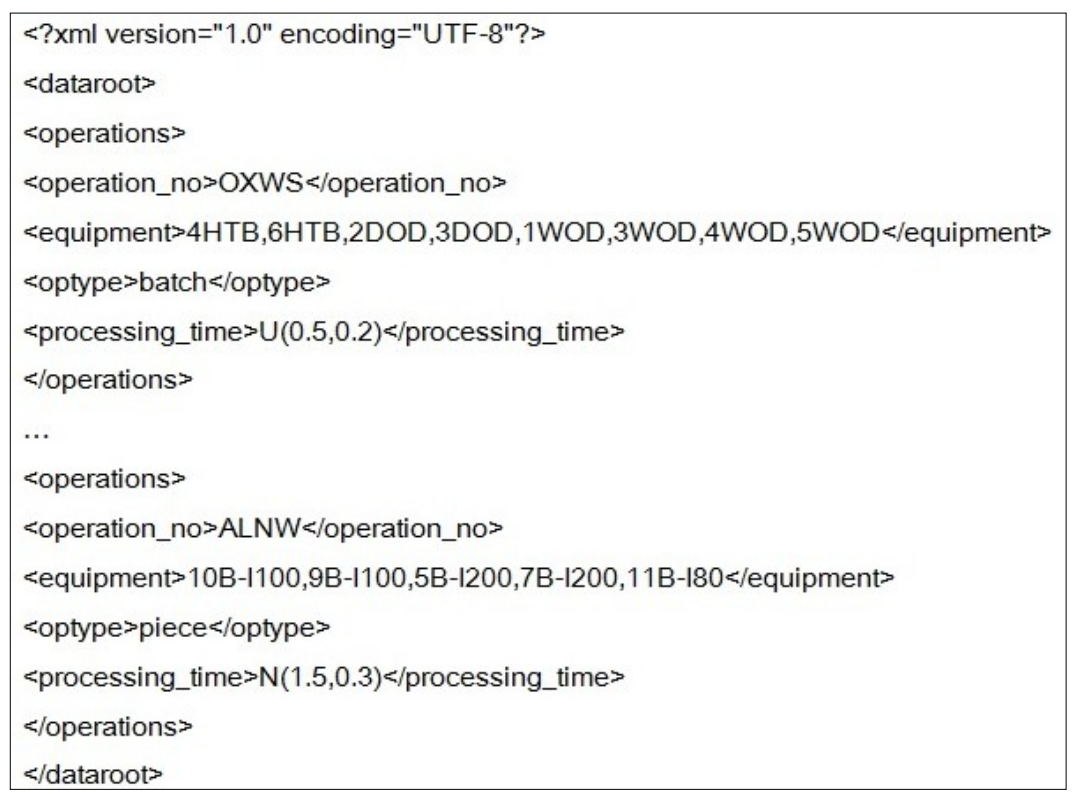

(5) Running simulation: In theory, CMfg helps run the simulation model of a wafer fab, i.e., running simulation as a cloud service (RSaaCS), in the following ways:

(i) Replicating the same simulation on several clouds: Since multiple simulation runs are usually required to obtain the average and standard deviations of the relevant attributes, they can be simultaneously run on several clouds, and then be aggregated to arrive at the final results.

(ii) Considering different possible values for uncertain/stochastic parameters: Many parameters in a fab simulation model are subject to uncertainties, so are usually represented using 
probability distributions. Different possible values of the same parameter can be taken into account at the same time by running the same simulation model on different clouds.

(iii) Evaluating the performances of different scheduling methods: Usually the effectiveness of a scheduling method has to be evaluated with the simulation model before it can be applied to the actual production system. In the proposed methodology, several scheduling methods can be simultaneously evaluated on different clouds to facilitate the comparison of these scheduling methods.

(iv) Partitioning the fab simulation model: As mentioned earlier, it usually takes a few hours to run a single replication of the fab simulation model. Such a problem can be tackled by dividing the fab simulation model into several smaller pieces that can be run on different clouds simultaneously and independently. Such a concept is illustrated in Figure 10. There are $m$ machines in this system. Originally, the system must be simulated as a whole. In order to enhance the efficiency of simulation, the system is divided into two parts-subsystem 1 (including machines $1 \sim p$ ) and subsystem 2 (including machines $p+1 \sim m$ ). To this end, the arrival time of each job to machine $p+1$ must be estimated, which then simulates the release time of the job to this machine. In this way, the two subsystems can be simulated at the same time. In a similar way, the system can be divided into $Q$ subsystems indicated with $q=1 \sim Q$ (see Figure 11). Subsystem $q$ involves machines $m(q-1) / Q+1 \sim m q / Q$. The arrival times of a job to these subsystems will be estimated when the job is released into the system. In the literature, there are a lot of methods for estimating the cycle time of a job, such as statistical analyses [21], soft computing applications [22,23], and hybrid approaches [24]. For a comprehensive literature review, refer to [25]. These methods can certainly be applied to estimate the step cycle time of a job. Then, the arrival time to a subsystem can be derived by adding the step cycle time of the previous subsystem to the release time.

Considering the degrees of difficulty and effects of these different treatments, RSaaCS can be summarized with a four-level pyramid (see Figure 12).

A well-known concept in cloud computing is RSC. The existing resource services are integrated into composite services to be invoked for addressing some complex manufacturing tasks. In summary, FSaaCS is a RSC of SAaaCS, EDaaCS, RCaaCS, FLaaCS, DRaaCS, RSaaCS, and other cloud services.

Figure 10. An example production system.

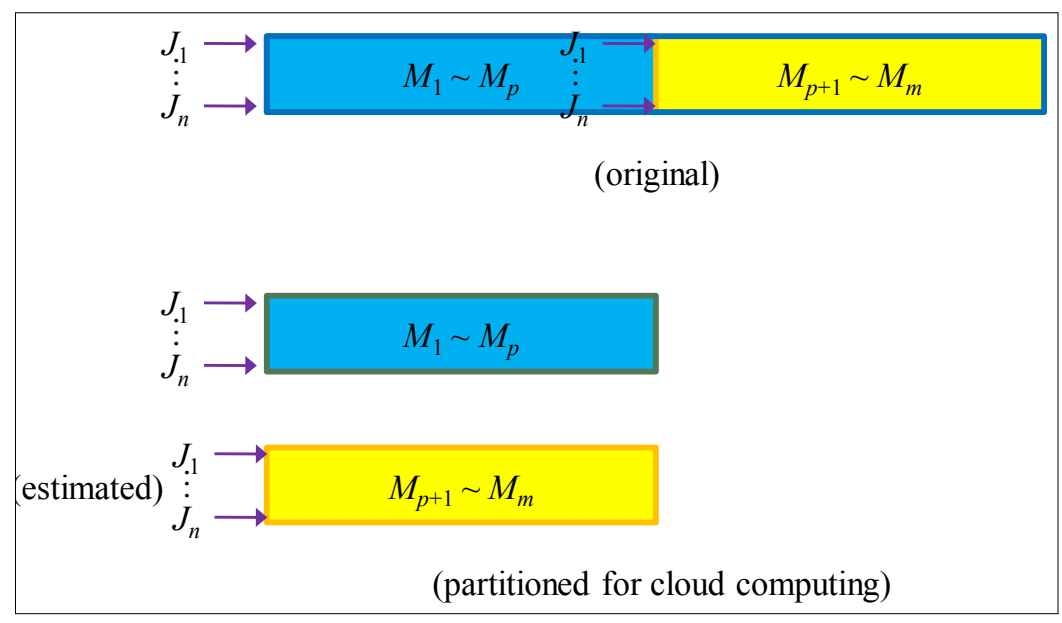


Figure 11. The arrival time to each subsystem is estimated when a job is released.

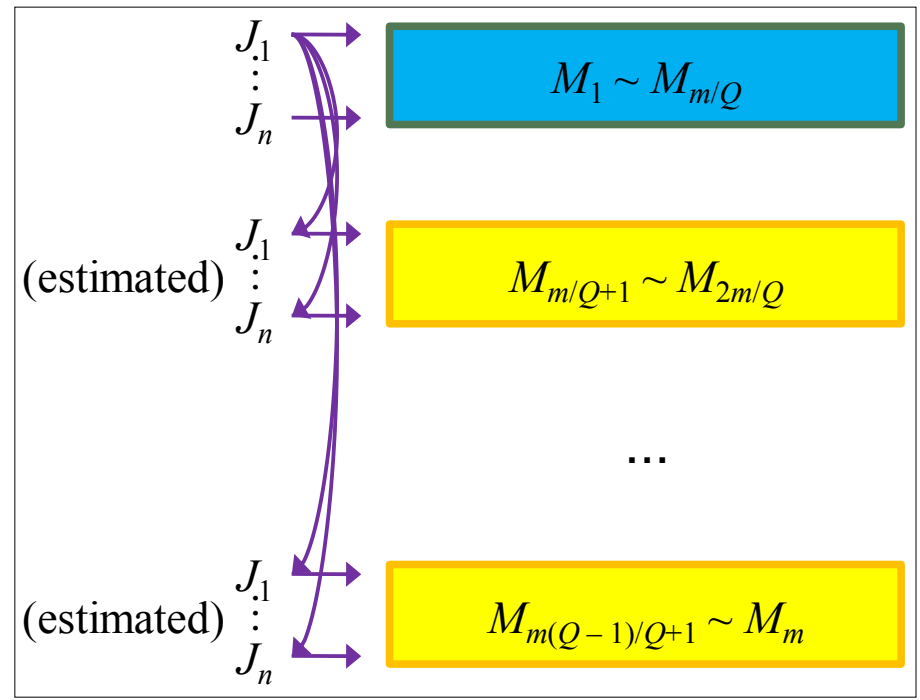

Figure 12. The four levels of recipe conversion as a cloud service (RCaaCS).

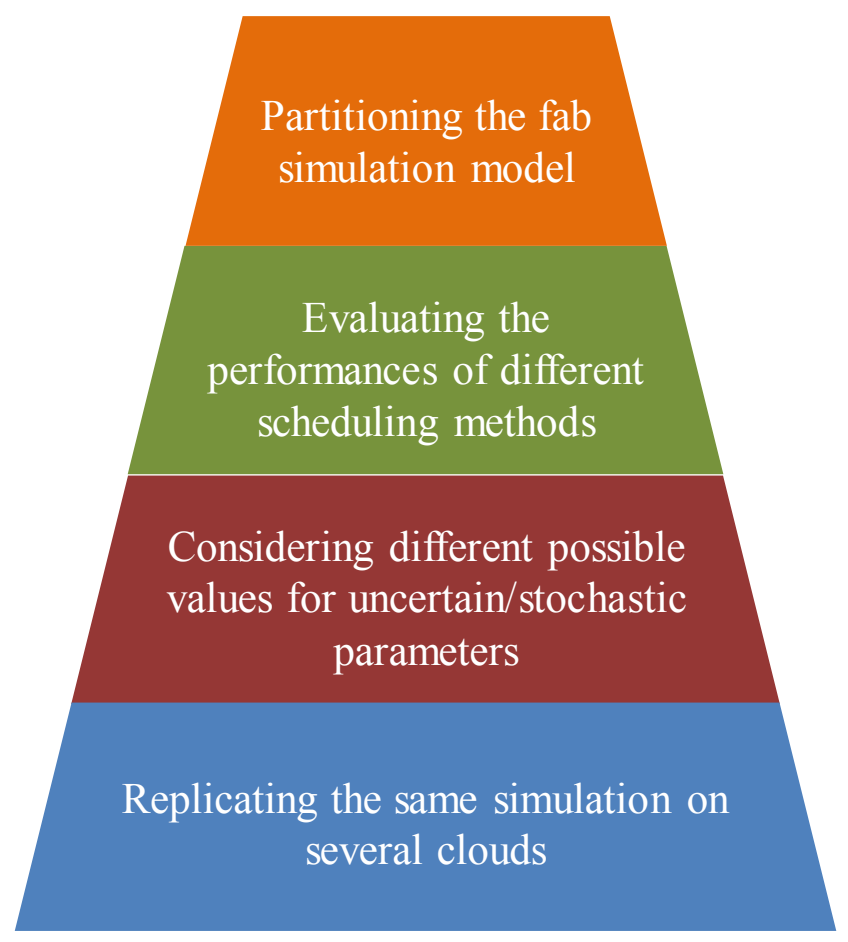

\subsection{Connection with the Competitiveness and Sustainability}

In various studies, simulation has been applied to evaluate the competitiveness or sustainability of an enterprise, or to formulate strategies to enhance the competitiveness or sustainability. For example, Miller [26] applied simulation to translate six-sigma philosophy, continuous flow manufacturing, and computer integrated manufacturing into competitive solutions that meet the objectives of a wafer fab. Zott [27] explored how the sustainability of a firm is linked to those of others within an industry using simulation. In Chen [13], fab simulation was applied to assess the effects of capacity reallocation on the yield competitiveness of a wafer fab. Improving the efficiency of fab simulation using CMfg services are certainly beneficial for the same purpose. 


\section{Conclusions and Future Research Directions}

As a potential solution to enhance the sustainable development of a semiconductor manufacturer, CMfg needs manufacturing-oriented insights to promote its practical applications in this industry. Manufacturing-oriented CMfg can be considered as the re-allocation of manufacturing resources with the aid of cloud computing technologies. To achieve this, this study first discussed the benefits of CMfg applications to a semiconductor manufacturer from the perspective of manufacturing. Such benefits were classified into the following five aspects: cost savings, efficiency, additional data analysis capabilities, flexibility, and closer partner relationships. Then, a SWOT analysis was done. The analysis results can guide a semiconductor manufacturer in planning CMfg implementation projects. Subsequently, as an innovative application of cloud computing in manufacturing, fab simulation is enhanced with cloud computing technologies in this study. In practice, a wafer fab encounters great difficulties in conducting the fab simulation, let alone to improve the efficiency and effectiveness of a fab simulation system.

After investigation, a series of CMfg services, including SAaaCS, EDaaCS, RCaaCS, FLaaCS, DRaaCS, and RSaaCS, have been proposed for assisting the corresponding fab simulation activities through the collaboration of cloud service providers, software vendors, equipment suppliers, and the wafer fab. The RSC of these CMfg services created an integrated CMfg service-FSaaCS.

The following are the main contributions to the body of knowledge:

(1) The applicability of CMfg to semiconductor manufacturing has rarely been discussed in the past. To this end, the results of the SWOT analysis performed in this study are worth considering.

(2) Production simulation has always been a tough task for a wafer fab, which inhibits the ability of further decision-making analyses based on the simulation model. This study discussed the CMfg service of fab simulation, which, if successfully applied, can increase the likelihood of solving some critical problems in managing a wafer fab, such as the elimination of dynamic bottlenecks.

The proposed methodology is a preliminary but workable architecture. The manufacturing-oriented architecture is quite clear, and has a high chance of being accepted. However, the proposed methodology must first go through an actual application to assess its advantages and disadvantages. In addition, the scalability of a wafer fab does not just mean a simple, quick way to expand the capacity, but also a convenient way to rent, resell, or dispose unused equipments. To these ends, the assistance from the equipment suppliers is essential. In other words, with equipment suppliers in the center, different semiconductor manufacturers can cooperate as a virtual capacity network to facilitate the migration of capacity among them, which needs to be further explored in future research.

\section{Acknowledgments}

This work was supported by National Science Council of Taiwan.

\section{Conflicts of Interest}

The author declares no conflict of interest. 


\section{References}

1. ACEsuppliers. Available online: http://ssttpro.acesuppliers.com/semiconductor_news/hi_tech_ enews_NewsId_6173.html (accessed on 11 November 2013).

2. The Liberty Times. Available online: http://www.libertytimes.com.tw/2013/new/sep/5/today-e12.htm (accessed on 11 November 2013).

3. Mell, P.; Grance, T. Perspectives on Cloud Computing and Standards; National Institute of Standards and Technology: Gaithersburg, MD, USA, 2009.

4. Li, B.H.; Zhang, L.; Wang, S.L. Cloud manufacturing: A service-oriented new networked manufacturing model. Comput. Integr. Manuf. Syst. 2010, 16, 1-9.

5. Xu, X. From cloud computing to cloud manufacturing. Robot. Comput. Integr. Manuf. 2012, 28, $75-86$.

6. Zhang, L.; Guo, H.; Tao, F.; Luo, Y.L.; Si, N. Flexible Management of Resource Service Composition in Cloud Manufacturing. In Proceedings of the 2010 IEEE International Conference on Industrial Engineering and Engineering Management, Macao, China, 7-10 December 2010.

7. Fan, Y.; Zhao, D.; Zhang, L.; Huang, S.; Liu, B. Manufacturing grid: Needs, concept and architecture. Lect. Notes Comput. Sci. 2004, 3032, 653-656.

8. Wu, D.; Greer, M.J.; Rosen, D.W.; Schaefer, D. Cloud manufacturing: Strategic vision and state-of-the-art. J. Manuf. Syst. 2013, 32, 564-579.

9. Cloud Sherpas. Available online: http:/www.cloudsherpas.com/cloud-services/manufacturing/ (accessed on 11 November 2013).

10. Epicor. End-to-end on-Demand ERP Solution for Manufacturers. Available online: http://www.epicor.com/Cloud/Pages/Cloud-Manufacturing.aspx (accessed on 11 November 2013).

11. Davidson, M. Why Cloud Manufacturing Software Adoption Rates Are Rising. Available online: https:/www.manufacturing.net/articles/2013/10/why-cloud-manufacturing-software-adoptionrates-are-rising (accessed on 11 November 2013).

12. Humphrey, A. SWOT Analysis for Management Consulting. Available online: http://www.sri.com/sites/default/files/brochures/dec-05.pdf (accessed on 11 November 2013).

13. Chen, T. Enhancing the yield competitiveness of a semiconductor fabrication factory with dynamic capacity re-allocation. Comput. Ind. Eng. 2009, 57, 931-936.

14. Chen, T.; Wu, H.C.; Wang, Y.C. Fuzzy-neural approaches with example post-classification for estimating job cycle time in a wafer fab. Appl. Soft Comput. 2009, 9, 1225-1231.

15. Chen, T. A flexible way of modelling the long-term cost competitiveness of a semiconductor product. Robot. Comput. Integr. Manuf. 2013, 29, 31-40.

16. Chen, T. A systematic cycle time reduction procedure for enhancing the competitiveness and sustainability of a semiconductor manufacturer. Sustainability 2013, 5, 4637-4652.

17. Makatsoris, C.; Leach, N.P.; Richards, H.D.; Besant, C.; Ristc, M. Addressing the Planning and Control Gaps in Semiconductor Virtual Enterprises. In IT and Manufacturing Partnerships; IOS Press: Amsterdam, The Netherlands, 1996.

18. Lean Manufacturing. Available online: http://wenku.baidu.com/view/ac8334707fd5360cbaladb21.html (accessed on 11 November 2013). 
19. Lin, E.; Minis, I.; Nau, D.S.; Regli, W.C. Virtual Manufacturing. Available online: http://www.isr.umd.edu/Labs/CIM/virtual.html (accessed on 11 November 2013).

20. Goldman, L.; Nagel, R.L.; Preiss, K. Agile Competitors and Virtual Organizations-Strategies for Enriching the Customer; Van Nostrand Reinhold: New York, NY, USA, 1995.

21. Tirkel, I. Cycle Time Prediction in Wafer Fabrication Line by Applying Data Mining Methods. In Proceedings of the 22nd Annual IEEE/SEMI Advanced Semiconductor Manufacturing Conference, New York, NY, USA, 16-18 May 2011.

22. Chen, T. A hybrid SOM-BPN approach to lot output time prediction in a wafer fab. Neural Process. Lett. 2006, 24, 271-288.

23. Chen, T. A FNP approach for evaluating and enhancing the long-term competitiveness of a semiconductor fabrication factory through yield learning modeling. Int. J. Adv. Manuf. Technol. 2009, 40, 993-1003.

24. Chen, T. An intelligent hybrid system for wafer lot output time prediction. Adv. Eng. Inform. 2007, 21, 55-65.

25. Chen, T. An effective fuzzy collaborative forecasting approach for predicting the job cycle time in wafer fabrication. Comput. Ind. Eng. 2013, 66, 834-848.

26. Miller, D.J. The Role of Simulation in Semiconductor Logistics. In Proceedings of the 1994 Winter Simulation Conference, Lake Buena Vista, FL, USA, 11-14 December 1994.

27. Zott, C. Dynamic capabilities and the emergence of intraindustry differential firm performance: Insights from a simulation study. Strateg. Manag. J. 2003, 24, 97-125.

(C) 2014 by the author; licensee MDPI, Basel, Switzerland. This article is an open access article distributed under the terms and conditions of the Creative Commons Attribution license (http://creativecommons.org/licenses/by/3.0/). 